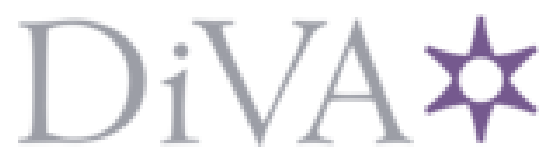

http://www.diva-portal.org

This is the published version of a paper published in American Naturalist.

Citation for the original published paper (version of record):

Wickman, J., Diehl, S., Blasius, B., Klausmeier, C A., Ryabov, A B. et al. (2017)

Determining Selection across Heterogeneous Landscapes: A Perturbation-Based Method and Its Application to Modeling Evolution in Space.

American Naturalist, 189(4): 381-395

https://doi.org/10.1086/690908

Access to the published version may require subscription.

N.B. When citing this work, cite the original published paper.

Permanent link to this version:

http://urn.kb.se/resolve?urn=urn:nbn:se:umu:diva-134209 


\title{
Determining Selection across Heterogeneous Landscapes: A Perturbation-Based Method and Its Application to Modeling Evolution in Space
}

\author{
Jonas Wickman, ${ }^{1, \star}$ Sebastian Diehl, ${ }^{2}$ Bernd Blasius, ${ }^{3}$ Christopher A. Klausmeier, ${ }^{4}$ \\ Alexey B. Ryabov, ${ }^{3}$ and Åke Brännström ${ }^{1,5}$ \\ 1. Integrated Science Lab, Department of Mathematics and Mathematical Statistics, Umeå University, SE-90187 Umeå, Sweden; 2. Integrated \\ Science Lab, Department of Ecology and Environmental Science, Umeå University, SE-90187 Umeå, Sweden; 3. Institute for Chemistry and \\ Biology of the Marine Environment, Carl von Ossietzky University Oldenburg, Carl-von-Ossietzky-Straße 9-11, D-26111 Oldenburg, Germany; \\ 4. W. K. Kellogg Biological Station and Department of Plant Biology, Michigan State University, Hickory Corners, Michigan 49060; 5. Evolution \\ and Ecology Program, International Institute for Applied Systems Analysis (IIASA), Schlossplatz 1, 2361 Laxenburg, Austria
}

Submitted July 5, 2016; Accepted November 15, 2016; Electronically published February 28, 2017

Online enhancements: appendixes.

\begin{abstract}
Aвstract: Spatial structure can decisively influence the way evolutionary processes unfold. To date, several methods have been used to study evolution in spatial systems, including population genetics, quantitative genetics, moment-closure approximations, and individualbased models. Here we extend the study of spatial evolutionary dynamics to eco-evolutionary models based on reaction-diffusion equations and adaptive dynamics. Specifically, we derive expressions for the strength of directional and stabilizing/disruptive selection that apply both in continuous space and to metacommunities with symmetrical dispersal between patches. For directional selection on a quantitative trait, this yields a way to integrate local directional selection across space and determine whether the trait value will increase or decrease. The robustness of this prediction is validated against quantitative genetics. For stabilizing/disruptive selection, we show that spatial heterogeneity always contributes to disruptive selection and hence always promotes evolutionary branching. The expression for directional selection is numerically very efficient and hence lends itself to simulation studies of evolutionary community assembly. We illustrate the application and utility of the expressions for this purpose with two examples of the evolution of resource utilization. Finally, we outline the domain of applicability of reaction-diffusion equations as a modeling framework and discuss their limitations.
\end{abstract}

Keywords: evolution, spatial, reaction-diffusion models, adaptive dynamics, quantitative genetics, metacommunities.

\footnotetext{
* Corresponding author; e-mail: jonas.wickman@umu.se.

ORCIDs: Diehl, http://orcid.org/0000-0002-3860-5051; Blasius, http://orcid.org /0000-0002-6558-1462; Ryabov, http://orcid.org/0000-0002-1595-6940.

Am. Nat. 2017. Vol. 189, pp. 381-395. (C) 2017 by The University of Chicago 0003-0147/2017/18904-57093\$15.00. All rights reserved. This work is licensed under a Creative Commons Attribution 4.0 International License (CC BY 4.0), which permits reuse of the work with attribution. DOI: $10.1086 / 690908$
}

\section{Introduction}

Heterogeneous landscapes provide both spatial variation in selective regimes and opportunities for geographic isolation. The notion that spatial heterogeneity should promote evolutionary diversification is therefore intuitively appealing. Although this intuition is sometimes foiled (Day 2001; Ajar 2003), several individual-based simulation and population genetics studies do indeed suggest that spatial structure can contribute to evolutionary branching and speciation (Felsenstein 1976; Doebeli and Dieckmann 2003; Servedio and Gavrilets 2004; Gavrilets and Vose 2005; Haller et al. 2013). However, understanding the interactions among spatial structure, population dynamics, and evolutionary dynamics has proved challenging. It is well recognized that the direction and magnitude of selection experienced by a population in a heterogeneous landscape depends both on the spatial pattern of local selection pressures and on the movement rates of individuals and genes across the landscape (Thompson 1999; Blondel et al. 2006). It is far from obvious under which circumstances this interplay of local selection, resulting from local ecological dynamics, and homogenizing dispersal results in directional selection, evolutionary stasis, or evolutionary diversification. It has, for example, been conjectured that mild geographical population structure may-paradoxically - be critical to the maintenance of evolutionary stasis at the species level over longer periods of time (Eldredge et al. 2005), in spite of widespread directional local selection (Kingsolver and Diamond 2011). Clearly, there is a need for a theory of evolution in space that can describe how spatially integrated selection in heterogeneous environments is driven by the interplay of local ecological dynamics and dispersal. 
The development of such a theory has proceeded along several venues. The perhaps earliest attempts trace back to diallelic one-locus models exploring the invasion of beneficial alleles and the evolution of polymorphisms in a continuous one-dimensional habitat (Fisher 1937, 1950; Haldane 1948; Slatkin 1973). Several extensions of these models have been made to include more than one locus (Slatkin 1975; Barton 1983), continuous polygenic characters (Slatkin 1978; Barton 1999), or density dependence and multidimensional space (Nagylaki 1975), among other examples. However, in these studies the ecological dynamics are typically very simple, and selection pressures are prescribed rather than derived from density- and frequencydependent interactions among phenotypes.

Conversely, models encompassing more realistic ecological interactions have typically neglected the underlying genetics altogether in favor of studying the evolution of phenotypes directly. A frequently employed approach has been to use spatially explicit, individual-based models that compute traitdependent reproduction and inheritance directly based on various rules (Doebeli and Dieckmann 2003; Gavrilets and Vose 2005; Mágori et al. 2005; Birand et al. 2012; Haller et al. 2013; Kubisch et al. 2014). These models exhibit potentially great ecological realism and have broad applicability but suffer from two limitations: they are computationally very demanding, and the results derived from these models are often difficult to analyze and check for robustness (but see the paragraph on moment-closure approximations in "Discussion" for further description and references).

Another approach to the inclusion of more realistic ecological interactions is to extend ecological models based on reaction-diffusion equations (a specific class of partial differential equations; see, e.g., Britton 1986; Holmes et al. 1994; Cantrell and Cosner 2004) to an evolutionary setting. Ecoevolutionary processes can then be studied using either a quantitative genetics (Lande 1979; Lande and Arnold 1983) or an adaptive dynamics (Metz et al. 1992; Dieckmann and Law 1996; Geritz et al. 1998) framework. The former accounts for standing variation in trait values and can be used to answer questions about the rate of evolutionary change and the degree of spatial variation of trait values within species caused by limited gene flow. These questions have already been touched on by Kirkpatrick and Barton (1997), Case and Taper (2000), and Norberg et al. (2012), among others. Adaptive dynamics, in turn, is concerned with mutation-driven evolution and is typically used to determine population-level selection gradients and such phenomena as evolutionary branching. These questions have received very little attention in the setting of spatial adaptive dynamics (but see, e.g., Mizera and Meszéna 2003; Troost et al. 2005). In particular, general mathematical expressions describing directional as well as stabilizing or disruptive selection have not yet been developed for reaction-diffusion models in continuous space. This is un- fortunate, as some questions - such as studying the direction of evolution of a trait in a spatially heterogeneous environment or how this spatial heterogeneity affects the potential for disruptive selection and subsequent diversification - can be easier to address in the reaction-diffusion adaptive dynamics framework than with the aforementioned approaches.

In this article, we develop central parts of an adaptive dynamics theory of evolution in space for populations whose ecological dynamics are well described by reaction-diffusion equations or certain metapopulation models. Specifically, we derive expressions for directional and stabilizing/disruptive selection acting on a quantitative phenotypic trait in organisms inhabiting a spatially heterogeneous landscape. These expressions are exact in the sense that they introduce no further approximations beyond the conceptual ones that underlie the use of adaptive dynamics and reaction-diffusion equations. Our effort serves two primary purposes. First, it enables us to draw general conclusions about the evolution of traits in such systems. Specifically, we determine how local selection pressures should be used in a weighted average across space to ascertain the population-level direction of selection of quantitative traits and show how this result can be understood in terms of reproductive value. We test that this result is consistent with a quantitative genetics model in the limit of small standing genetic variation. We also show that spatial structure always contributes to disruptive selection in the absence of directional selection. Second, the derived expressions enable efficient numerical investigation of spatial evolutionary dynamics in eco-evolutionary systems described by reaction-diffusion equations. We illustrate the application and utility of the developed techniques in two examples of the evolution of resource utilization in a heterogeneous environment. Finally, we discuss the limitations of reaction-diffusion equations as a modeling framework and delineate the range of eco-evolutionary systems over which they are applicable.

\section{Evolutionary Dynamics in Spatially Structured Populations}

Imagine a population inhabiting a heterogeneous landscape over which selection pressures for a quantitative trait, such as the size of a body part, varies. In certain regions selection favors trait values larger than the current population average, while in others it favors smaller trait values. Will selection result in an increase or a decrease in the mean trait value across the population? The intuitive approach of simply averaging selection across all members of the populations gives the wrong result. Below we show that one needs to take a particular weighted average with a disproportionate contribution from highly populated areas. We demonstrate that the expression for directional selection is valid in the frameworks of both adaptive dynamics and quantitative genetics, provided that standing genetic variation is small. 
Invasion Fitness in Spatially Structured Populations

Before we start our analysis of selection in reaction-diffusion equations, we present a brief summary of adaptive dynamics theory (Metz et al. 1992; Dieckmann and Law 1996; Geritz et al. 1998), which is a framework for studying evolutionary processes by calculating the so-called invasion fitness of a rare mutant phenotype in a community of one or more resident phenotype(s) at equilibrium. This invasion fitness is the long-term per capita growth rate of the mutant while the mutant is still rare compared with the resident(s) and is thus a measure of the mutant's ability to cope with the environment set by the resident(s) while its own influence on the environment is still negligible. Write $A_{\mathrm{r}}$ and $A_{\mathrm{m}}$ for the densities of a resident and a mutant phenotype, respectively, and let $\chi_{\mathrm{r}}$ and $\chi_{\mathrm{m}}$ be traits that uniquely characterize each of the two phenotypes (we shall throughout the text use subscripts " $\mathrm{r}$ " and " $\mathrm{m}$ " for quantities pertaining to residents and mutants, respectively). In a spatially unstructured system, the growth of the resident population is governed by

$$
\frac{\mathrm{d} A_{\mathrm{r}}}{\mathrm{d} t}=G\left(E_{\mathrm{r}}, \chi_{\mathrm{r}}\right) A_{\mathrm{r}},
$$

where the net per capita growth rate $G$ depends on the environment set by the resident, $E_{\mathrm{r}}=E\left(\chi_{\mathrm{r}}\right)$, and on the resident's trait value. When the resident is at equilibrium, with density $A_{\mathrm{r}}^{*}$ setting an equilibrium environment $E_{\mathrm{r}}^{*}$, and the mutant is still rare enough to not affect the environment, the mutant's growth rate can be expressed as

$$
\frac{\mathrm{d} A_{\mathrm{m}}}{\mathrm{d} t}=G\left(E_{\mathrm{r}}^{*}, \chi_{\mathrm{m}}\right) A_{\mathrm{m}} .
$$

The above equation is a linear, first-order, ordinary differential equation. The solution is

$$
A_{\mathrm{m}}(t)=c \exp \left(G\left(E_{\mathrm{r}}^{*}, \chi_{\mathrm{m}}\right) t\right)
$$

where $c$ is a constant representing the initial density of the mutant. This solution describes the invasion well as long as the mutant remains rare relative to the resident. From this, we see that the mutant density will increase if $G$ is positive and will decline if $G$ is negative. Thus, $G\left(E_{\mathrm{r}}^{*}, \chi_{\mathrm{m}}\right)$, the exponential growth rate of the mutant while still rare, is considered the invasion fitness of the mutant. For a given resident trait, we can think of $G$ as being a function of the trait value $\chi$ and calculate the directional selection acting on a resident phenotype by computing how invasion fitness changes with changes in the trait value. In adaptive dynamics, the selection gradient, defined as

$$
D\left(\chi_{\mathrm{r}}\right)=\left.\frac{\partial G\left(E_{\mathrm{r}}^{*}, \chi\right)}{\partial \chi}\right|_{\chi=\chi_{\mathrm{r}}},
$$

is the measure of the strength and direction of this directional selection.
In a spatially heterogeneous system, the density of a spatially structured population depends on both time and space and can be denoted by $A(t, \mathbf{x})$, where $\mathbf{x}=$ $\left(x_{1}, \ldots, x_{n}\right)$ describes the location in $n=1,2$, or 3 spatial dimensions. We assume that the ecological dynamics can be described by a partial differential equation:

$$
\frac{\partial A(t, \mathbf{x})}{\partial t}=F(E(\chi), \mathbf{x}, \chi) A .
$$

Here the local rate of change is described by a differential operator $F$, which typically not only depends on the local population density $A(\mathbf{x})$, the spatial coordinate $\mathbf{x}$, and the trait under selection, $\chi$, but also contains spatial derivatives describing passive transport or active movement in space. One important class of such systems are reaction-diffusion systems, where the differential operator takes the form

$$
F(E(\chi), \mathbf{x}, \chi) A=G(E(\chi), \mathbf{x}, \chi) A+d(\chi) \Delta A .
$$

Here the function $G$ describes the density-dependent net growth of the phenotypes at different points in space (mediated through their effect on the environment $E$ ), $d$ is the diffusivity, and $\Delta A=\partial^{2} A / \partial x_{1}^{2}+\cdots+\partial^{2} A / \partial x_{n}^{2}$ is the Laplacian of $A$, which describes the random movement or transport of individuals from more to less densely populated locations in all $n$ directions. When we derive in the next section an expression for the selection gradient in heterogeneous space, we initially consider only such random (diffusive) movement. Further down, in example 2, we extend the approach to include constant directional movement (advection).

Much like the nonspatial case, considering the case of a mutant that is initially very rare results in a linear partial differential equation describing the mutant's population growth:

$$
\frac{\partial A_{\mathrm{m}}}{\partial t}=F\left(E_{\mathrm{r}}^{*}, \mathbf{x}, \chi_{\mathrm{m}}\right) A_{\mathrm{m}} .
$$

This equation cannot be solved in the same way as the ordinary differential equation of the nonspatial case, since $F$ contains partial spatial derivatives. There is, however, by the theory of linear differential equations an expression for the solutions:

$$
A_{\mathrm{m}}(\mathbf{x}, t)=\sum_{i=0}^{\infty} c_{i} e^{\lambda_{i} t} A_{i}(\mathbf{x}) .
$$

Each term in the above sum is a different possible solution to equation (7), where each solution assumes a specific (temporally constant) shape of the spatial density distribution of individuals $A_{i}(\mathbf{x})$, while the total density of individuals is initially determined by $c_{i}$ and subsequently increases or decreases over time at an exponential rate determined by the exponent $\lambda_{i}$. The functions $A_{i}(\mathbf{x})$ and numbers $\lambda_{i}$ 
are the so-called eigenfunctions and eigenvalues of the operator $F$ and solve the equation $F A_{i}(\mathbf{x})=\lambda_{i} A_{i}(\mathbf{x})$. The general solution, equation (8), emerges from the combined contributions of these specific solutions. Eventually, the term with the largest $\lambda_{i}$ will dominate and determine the long-term exponential growth rate of the mutant population. This quantity, known as the dominant eigenvalue, is written $\lambda_{\mathrm{d}}$ and is the natural generalization of invasion fitness for a mutant in a spatially structured system.

\section{Perturbation-Based Method for Calculating Selection across Heterogeneous Space}

Having introduced the concept of invasion fitness for spatially structured populations, we next derive expressions for disruptive/stabilizing selection in populations that follow reaction-diffusion dynamics. The approach rests on the assumption in adaptive dynamics that single mutations have small phenotypic effects. Hence, the selection gradient is essentially describing first-order, or weak, selection. This is not in itself a new approach (especially for matrix models; see, e.g., van Baalen and Rand 1998; Caswell 2001; Rousset 2004), but by using perturbation theory for operators describing reaction-diffusion dynamics in systems with absorptive, reflective, or periodic boundaries we are able to compute an expression for the selection gradient that does not involve any explicit computations of eigenvalues or vectors. This has two major benefits. First, it allows us to evaluate selection only on the basis of the ecological equilibrium dynamics, which means that some immediate, general conclusions can be drawn regarding the direction in which a trait will evolve by averaging local selection gradients. Second, it provides great savings in terms of numerical computations of selection gradients when simulating discretized versions of evolutionary reaction-diffusion systems. This approach likewise yields some new insight into how environmental heterogeneity contributes to disruptive selection and how diversification is affected by this additional disruptive selection.

Perturbation Theory Can Be Used to Determine Directional Selection. If an unknown differential operator can be expressed as the sum of a known operator plus some small disturbance, it is often possible to calculate approximations of the unknown operator. For instance, we know that the eigenvalue of the operator $F$ of equation (7) must be 0 when it is operating on the resident phenotype at ecological equilibrium, since the equilibrium density by definition will neither grow nor decline. As such, equation (7) will be

$$
0=\frac{\partial A_{\mathrm{r}}^{*}}{\partial t}=F A_{\mathrm{r}}^{*}
$$

In a reaction-diffusion system like equation (6), knowing that the operator acting on the equilibrium density of the resident has a dominant eigenvalue of 0 , we can perform perturbation calculations to derive an expression for the selection gradient $D\left(\chi_{\mathrm{r}}\right)$ for a resident phenotype in ecological equilibrium (for details, see app. A; apps. A-G are available online):

$$
\begin{gathered}
D\left(\chi_{\mathrm{r}}\right)=\frac{1}{\int A_{\mathrm{r}}^{* 2} \mathrm{~d} \mathbf{x}} \\
\times\left(\left.\int A_{\mathrm{r}}^{*_{2}} \frac{\partial G\left(E_{\mathrm{r}}^{*}, \mathbf{x}, \chi\right)}{\partial \chi}\right|_{\chi_{\mathrm{r}}} \mathrm{d} \mathbf{x}-\left.\frac{\partial d(\chi)}{\partial \chi}\right|_{\chi_{\mathrm{r}}} \int\left|\nabla A_{\mathrm{r}}^{*}\right|^{2} \mathrm{~d} \mathbf{x}\right) .
\end{gathered}
$$

Equation (10) describes the selection gradient for a resident phenotype with equilibrium density $A_{\mathrm{r}}^{*}$ in the equilibrium environment $E_{\mathrm{r}}^{*}$ set by the resident; $\partial G\left(E_{\mathrm{r}}^{*}, \mathbf{x}, \chi\right) /\left.\partial \chi\right|_{\chi_{\text {. }}}$ describes how per capita growth changes and $\partial d(\chi) /\left.\partial \chi\right|_{\chi_{t}}$ how diffusivity changes with changing trait value. The integral is over the entire available space. The gradient $\nabla A_{\mathrm{r}}^{*}$ is a vector describing the slope of the resident's density distribution in each spatial direction, and $\left|\nabla A_{\mathrm{r}}^{*}\right|$, the Euclidian vector norm of $\nabla A_{\mathrm{r}}^{*}$, is the maximum rate of change, which is always in the direction of the gradient.

From equation (10) we can draw general conclusions about directional selection in spatial systems. First, if diffusivity is trait independent-that is, if $\partial d / \partial \chi=0$ - then equation (10) simplifies to

$$
D\left(\chi_{\mathrm{r}}\right)=\left.\frac{1}{\int A_{\mathrm{r}}^{* 2} \mathrm{~d} \mathbf{x}} \int A_{\mathrm{r}}^{* 2} \frac{\partial G\left(E_{\mathrm{r}}^{*}, \mathbf{x}, \chi\right)}{\partial \chi}\right|_{\chi_{\mathrm{r}}} \mathrm{d} \mathbf{x} .
$$

In the complete absence of diffusion, $\partial G\left(E_{\mathrm{r}}^{*}, \mathbf{x}, \chi\right) /\left.\partial \chi\right|_{\chi_{\mathrm{r}}}$ is the local selection gradient at each point in space. The selection gradient acting on the population of the resident phenotype as a whole (described by eq. [11]) can thus be interpreted as a weighted average of local selection gradients. The weights are $A_{\mathrm{r}}^{* 2}$, meaning that the contributions to the selection gradient at points in space where the resident is abundant are disproportionately stronger than where it is rare. This squared term can be understood by analogy to a classical result for sensitivity analysis of matrix models, where the sensitivity of growth rate depends on both the right and the left eigenvector, corresponding respectively to the stable population distribution and the individual reproductive value (Caswell 2001). In the reaction-diffusion equations we investigate, these right and left eigenvectors are identical due to the spatial symmetry of dispersal. Each is given by $A^{*}(\mathbf{x})$, resulting in the squared weighting term in equation (11) (app. A).

Second, if instead the trait does not affect local fitness but only the dispersal rate- that is, if $\partial G / \partial \chi=0$ - equation (10) simplifies to 


$$
D\left(\chi_{\mathrm{r}}\right)=-\left.\frac{1}{\int A_{\mathrm{r}}^{* 2} \mathrm{~d} \mathbf{x}} \frac{\partial d(\chi)}{\partial \chi}\right|_{\chi_{\mathrm{r}}} \int\left|\nabla A_{\mathrm{r}}^{*}\right|^{2} \mathrm{~d} \mathbf{x} .
$$

Two conclusions directly follow. First, since $\left|\nabla A_{\mathrm{r}}^{*}\right|$ is the local slope of the resident's density distribution, selection on diffusivity depends on the degree to which the resident is heterogeneously distributed in the landscape. Hence, if the distribution of the resident is completely homogeneous, then the selection gradient will be 0 . Second, since both $\int A_{\mathrm{r}}^{* 2} \mathrm{~d} \mathbf{x}$ and $\int\left|\nabla A_{\mathrm{r}}^{*}\right|^{2} \mathrm{~d} \mathbf{x}$ are always positive, selection will always be for lower diffusivity, which is in line with the findings of earlier investigations by Hastings (1983) and Dockery et al. (1998).

Equation (10) is valid for reaction-diffusion equations with periodic, absorptive, and reflective boundary conditions or some combination thereof. In appendixes $\mathrm{A}$ and $\mathrm{E}$, this is extended to a larger class of systems.

Sympatric and Parapatric Sources of Disruptive Selection Can Be Distinguished. When directional selection ceases and the selection gradient is 0 , the resident is at what is known as an evolutionarily singular point. Here the second derivative of invasion fitness with respect to a mutant's trait value must be determined to evaluate whether the evolutionarily singular point is a fitness maximum (evolutionarily stable strategy), at which no more evolution will occur, or a fitness minimum, at which a phenotype will split into two. A full account of selection at an evolutionarily singular strategy is given in appendix B. Here we present the three most important conclusions that arise from this analysis.

First, all other things equal, spatial heterogeneity in local selection regimes always promotes evolutionary branching and diversification. The reason is that the possibility for the mutant to assume other spatial distributions than the resident's always contributes positively to the second derivative of its invasion fitness (eq. [5]).

Second, by splitting the equation for disruptive/stabilizing selection (eq. [5]) into two terms, two cases of evolutionary branching can be distinguished that we with a slight abuse of terminology call sympatric and parapatric diversification. In the first case, when the primary source of disruptive selection is the spatially averaged ecological dynamics, a phenotype can branch into two phenotypes with exactly the same spatial distribution as their progenitor. In the second case, when the only source of disruptive selection is the environmental heterogeneity - that is, spatial variation in directional selection - at least one of the new phenotypes must have a spatial distribution different from that of the progenitor. The second type of branching may occur even if the spatially averaged dynamics contribute stabilizing selection, such as in example 1 below.
Third, if the diffusion rate is not under selection we can in many situations (for details, see app. B) estimate an upper limit to the amount of disruptive selection (i.e., the second derivative of the invasion fitness with respect to the mutant trait) that can be contributed by environmental heterogeneity as

$$
2 K \frac{\left(1 / \int A^{*_{2}} \mathrm{~d} \mathbf{x}\right) \int A^{*_{2}}\left[\left.(\partial G / \partial \chi)\right|_{\chi}=\chi_{\mathrm{r}}\right]^{2} \mathrm{~d} \mathbf{x}}{d}
$$

Here $K$ is a constant that depends only on the shape and size of the landscape. The numerator can be interpreted as the weighted variance of local selection gradients, in the same spirit as in equation (11). This means that the maximal amount of disruptive selection coming from environmental heterogeneity for any given landscape depends on the ratio between the overall variability in local directional selection regimes and diffusion rate $d$.

Results concerning Directional Selection Are Also Valid in a Spatial Quantitative Genetics Setting in the Limit of Low Standing Variation. In a nonspatial setting, it has been noted that there are some strong similarities between adaptive dynamics and quantitative genetics (Waxman and Gavrilets 2005). We investigate to what extent our conclusions about directional selection derived in the setting of adaptive dynamics also hold true in a spatial quantitative genetics setting by using a model developed by Kirkpatrick and Barton (1997) that describes trait evolution in a population with normally distributed trait values at each point in space. We conclude that, in the limit of small standing genetic variation, the quantitative genetics model reduces to a reaction-diffusion equation for the ecological dynamics and that trait evolution becomes completely determined by the same expression for directional selection that we derived in the adaptive dynamics context. The details can be found in appendix C.

\section{Application of the New Method to Evolutionary Community Assembly in Continuous Space and in Metacommunities: A General Recipe and Two Examples}

An important application of the new method for calculating selection across heterogeneous space is to the study of evolutionary community assembly. Since equation (10) makes it possible to efficiently calculate the direction and magnitude of selection acting on a set of resident populations, methods such as setting the rate of change of a trait to be proportional to the selection gradient can be used to investigate the coevolutionary dynamics of ecological communities. Many methods for studying evolutionary community assembly and for numerically implementing the equations of the previous section exist. Below we describe one such combination of methods that is applicable both to continuous space and to landscapes of discrete habitat patches occupied by metacommunities. 

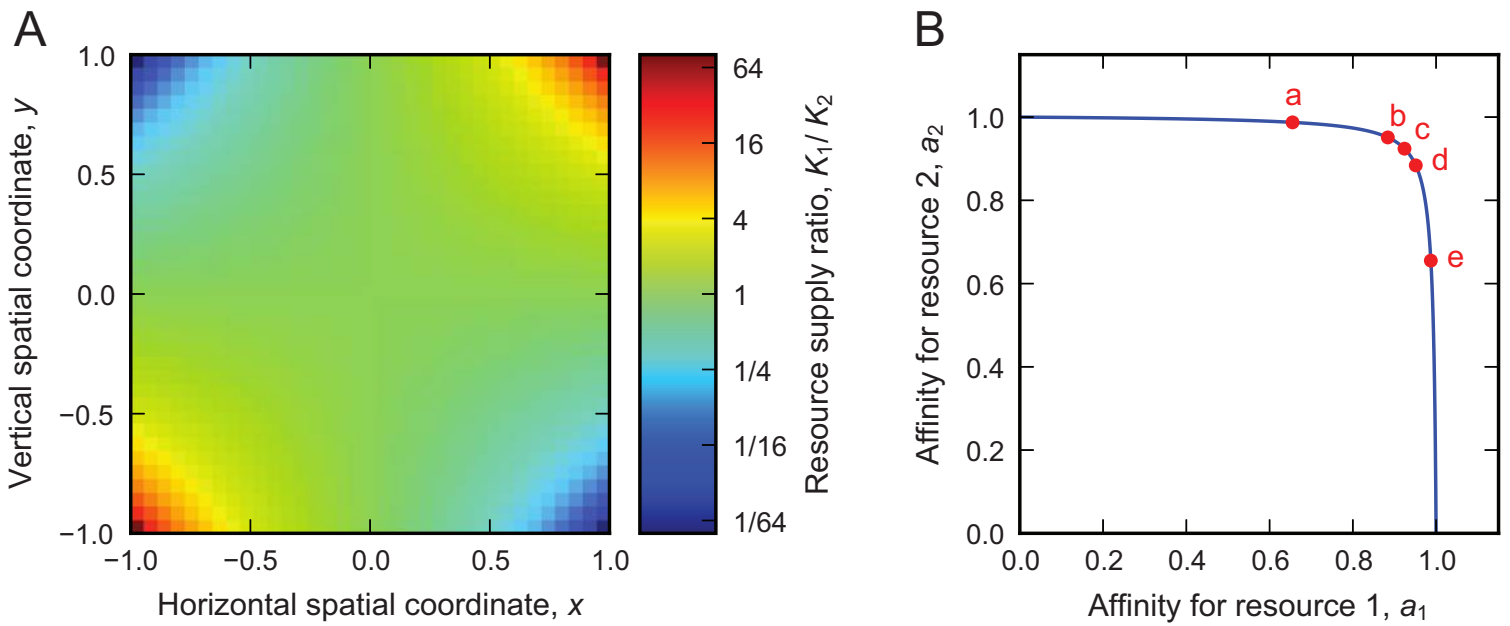

Figure 1: Example 1. A, Resource supply landscape. The ratio $K_{1} / K_{2}$ of the maximum resource densities of the two resources is distributed as two crossed saddle shapes in space. The sum of the maximum resource densities of the resources is constant so that $K_{1}+K_{2}=2.05$. $B$, Trade-off curve describing the relationship between consumers' resource affinities for the two resources. The concave-down shape of the curve implies that the trade-off is weak and imposes a cost to specialization. Evolution of the trait $\chi$ is constrained to yield resource affinity combinations on the trade-off curve. The red dots indexed by $a-e$ indicate the resource affinity of consumers $a-e$ in figure 2 .

\section{Evolutionary Assembly in Continuous Space}

For numerical implementation we discretize (continuous) space into a lattice (as in fig. $1 A$ ). This reduces the partial differential equation (6) to a large set of coupled ordinary differential equations, the density distribution $A(\mathbf{x})$ to a vector of densities $\mathbf{A}$, and the differential operator $F$ to a square matrix. The discretized equation system is then integrated to ecological equilibrium. The invasion fitness of a mutant in the system at a given ecological equilibrium is then found by computing the dominant eigenvalue of the matrix $F$, and an expression analogous to equation (10) can be derived (see app. D).

The evolutionary assembly process is computed in three principal steps. First, during evolutionary community assembly selection will be directional for most of the time, and several methods can be used to compute the evolutionary response to directional selection. For instance, to produce figure $2 A$ in example 1 below, we set the rate of change of a trait to be proportional to the selection gradient, that is, $\mathrm{d} \chi_{\mathrm{r}} / \mathrm{d} t=\epsilon D\left(\chi_{\mathrm{r}}\right)$, where $\epsilon$ is a small number separating ecological and evolutionary timescales; $\epsilon$ was chosen to be sufficiently small for the ecological dynamics to be very close to equilibrium at all times. This type of gradient dynamics is commonly used in computing the effects of directional selection and has the same general form as, for example, the canonical equation of adaptive dynamics (Dieckmann and Law 1996; Champagnat 2003) or the Lande equation (Lande 1979). For figure 3 in example 1 we were interested only in finding the eco-evolutionary equilibria, and hence we used the secant method (see, e.g., Press et al. 2007) with a bound on step size to find where the selection gradient for each resident was 0 .

Second, when directional selection ceases and an evolutionarily singular point is reached, direct numerical computations of the dominant eigenvalue of the matrix $F$ for trait values close to each resident are used to determine whether each resident phenotype is at an evolutionary branching point or an evolutionarily stable strategy. If any of the residents is at an evolutionary branching point, a portion of its density is split off to form a new phenotype with a trait value close to the one of the branching resident, and further coevolution of the new ensemble is calculated as described above. Third, once all phenotypes are at an evolutionarily stable strategy, further trait values with positive invasion fitness may still exist. In such cases, a mutant with a trait value at the global maximum in the fitness landscape is allowed to invade the ensemble. As this requires large mutational steps, it is done by direct numerical calculation of the invasion fitness, that is, the dominant eigenvalue of $F$. The described three steps of the evolutionary assembly process are then repeated until no more positive invasion fitness is available. An example of the entire process (fig. $2 A$ ) and the resulting fitness landscape (fig. $2 B$ ) is illustrated in example 1 below.

\section{Evolutionary Assembly in Metacommunities}

Our new method for the calculation of selection across a heterogeneous, but continuous, landscape is easily adapted to the setting of an evolving metacommunity on a set 

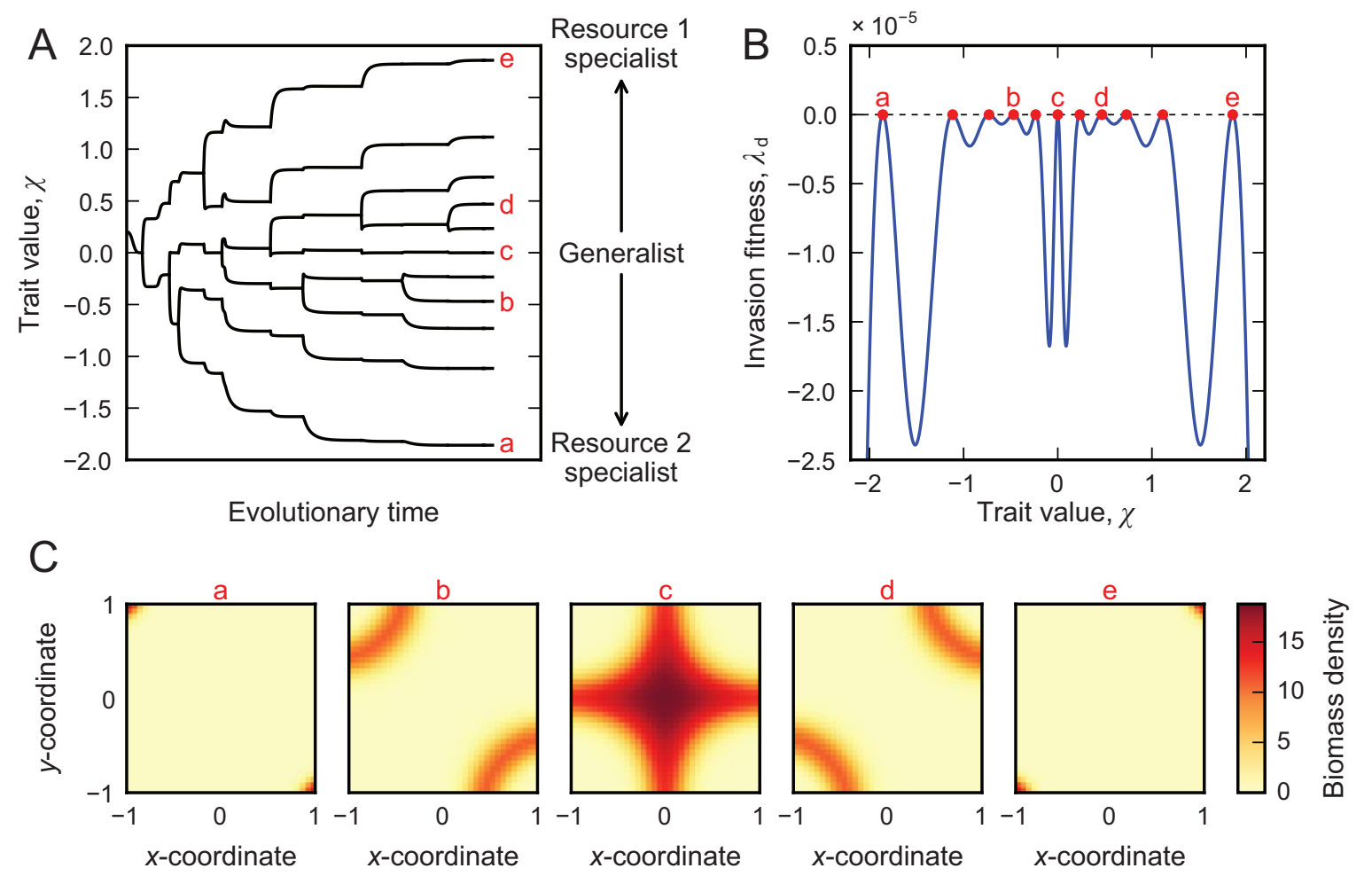

Figure 2: A, Example of trait evolution on the resource supply rate landscape in figure 1, with parameters as in table 1. Consumers continually evolve until an eco-evolutionary equilibrium is reached at the final time point. Letters $a-e$ indicate which branch corresponds to which consumer density distribution in $C$. $B$, Fitness landscape at the final point in time of $A$, showing that all consumers reside on local fitness maxima, with no further invasions possible. Red dots indicate resident consumers' trait values. $C$, Equilibrium distribution in space of the five consumers indicated by letters $a-e$ in $A$ and $B$. Consumers with positive trait values are resource 1 specialists, consumers with negative trait values are resource 2 specialists, and consumers with trait value $0(c)$ are generalists and have equal affinity for both resources. Darker shading indicates higher consumer density. Although pictured separately for clarity, there is significant spatial overlap among consumers, and several consumers can co-occur at the same spatial coordinate.

of discrete patches, where the dynamics are governed by coupled ordinary differential equations. Since the justdescribed numerical implementation of continuous-space scenarios is technically equivalent to the metacommunity formalism, we do not treat the latter any further in the main text but refer the reader instead to appendix D. There we develop in detail a formalism for the study of community assembly in an evolving metacommunity, where both local growth rates in different patches and dispersal rates between patches may depend on a selected trait of the evolving phenotypes. Additionally, in appendix $\mathrm{C}$ the results concerning the spatial quantitative genetics model of the previous section are restated for a metapopulation model on discrete patches.

In the following sections we illustrate the application of our perturbation-based method to the study of evolution in heterogeneous space with two examples of systems where consumers compete for two limiting resources along spatial gradients and experience an evolutionary trade-off in the ef- ficiency with which they can use the two resources. Example 1 is crafted as an easy-to-understand, heuristic case study assuming a highly regular, two-dimensional geometry of resource supply and exclusively random (diffusive) dispersal of consumers. Example 2 is a much more realistic case study of phytoplankton competing for nutrients and light along opposite vertical gradients and includes the additional complication of directed movement (which requires a variable transformation, described in app. E). It also shows the relative ease with which an existing ecological study can be extended to include evolutionary dynamics. In both examples we focus on the most relevant and challenging issue in a spatial context, that is, the influence of the speed of (diffusive or directed) movement across heterogeneous space on evolutionary outcomes. In addition, we evaluate the computational efficiency of the perturbation techniques. The reader interested in learning to apply the method may also consult appendix F, in which a discrete example simple enough to solve analytically is treated. 


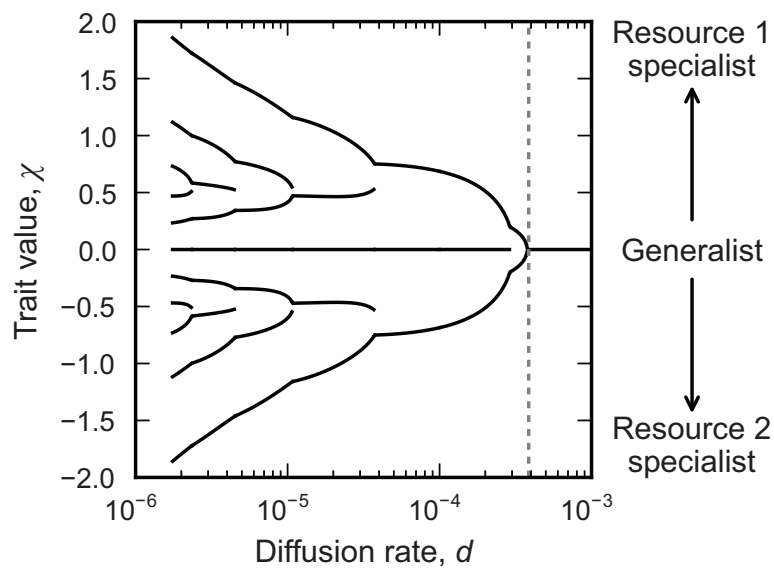

Figure 3: Example 1: distribution of consumer trait values at evolutionary equilibrium for different rates of diffusion. When the rate of diffusion is high, a generalist can monopolize the entire space, whereas low rates leave room for many coexisting consumers. The vertical dashed line indicates the upper limit for disruptive selection $\left(d=3.86 \cdot 10^{-4}\right)$, as calculated by equation (18). Parameters other than $d$ are as in table 1 . See appendix $\mathrm{G}$ for an account of the nature of the bifurcations and why the diagram has gaps.

\section{Example 1: Randomly Dispersing Consumers Competing} for Resources with Spatially Variable Supply Rates

In our first example, randomly dispersing consumers compete for two heterogeneously distributed resources on a square landscape (with spatial coordinates $x$ and $y$ ). Consumers take up resources at the coordinate at which they reside and move randomly through the landscape as described by a diffusion process. The two resources are substitutable, renew locally at each coordinate, and do not disperse. As an example, we envision plants consuming two substitutable nutrients (e.g., nitrate and ammonia) and dispersing seeds randomly, but the scenario is applicable to other systems with primarily locally renewed resources (e.g., randomly dispersing herbivores consuming substitutable plants). The dynamics at each point in space of the density $A_{k}(t, x, y)$ of consumer $k$ and of the two resource densities $R_{1}(t, x, y)$ and $R_{2}(t, x, y)$ are described by the following equations:

$$
\begin{aligned}
\frac{\partial A_{k}}{\partial t} & =G\left(R_{1}, R_{2}\right) A_{k}-m A_{k}+d \Delta A_{k}, \\
\frac{\mathrm{d} R_{1}}{\mathrm{~d} t} & =r_{1}\left(K_{1}-R_{1}\right)-\sum_{k} G_{\max } \frac{a_{1 k} R_{1}}{1+a_{1 k} R_{1}+a_{2 k} R_{2}} A_{k}, \\
\frac{\mathrm{d} R_{2}}{\mathrm{~d} t} & =r_{2}\left(K_{2}-R_{2}\right)-\sum_{k} G_{\max } \frac{a_{2 k} R_{2}}{1+a_{1 k} R_{1}+a_{2 k} R_{2}} A_{k} .
\end{aligned}
$$

Consumers take up resources 1 and 2 and convert them into growth according to a function derived from the concept of the "synthesizing unit" (Kooijman 1998) at a maximal rate $G_{\max }$. For the case of two substitutable resources, this yields

$$
G\left(R_{1}, R_{2}\right)=G_{\max } \frac{a_{1 k} R_{1}+a_{2 k} R_{2}}{1+a_{1 k} R_{1}+a_{2 k} R_{2}},
$$

where $a_{1 k}$ and $a_{2 k}$ are consumer $k$ 's affinities for resources 1 and 2 . In the limiting case of only one resource being present, this function reduces to the familiar Monod function (Monod 1949) with $1 / a_{1 k}$ or $1 / a_{2 k}$, respectively, being halfsaturation constants.

In the absence of consumers, resources 1 and 2 follow semichemostat dynamics with turnover rates $r_{1}$ and $r_{2}$ and maximum resource densities at each point in space $K_{1}(x, y)$ and $K_{2}(x, y)$. Semichemostat dynamics are commonly used to describe the renewal of abiotic resources such as mineral nutrients (Tilman 1982) and ensure, for the nonspatial case of two resources, that the system settles to a locally stable ecological equilibrium. Although not guaranteed for the spatial case, we observe only stable equilibrium dynamics for the range of parameters explored in this example.

For ease of interpretation, we assume a highly regular geometry of the resource supply landscape. Specifically, total resource supply is constant $\left(K_{1}+K_{2}=2.05\right.$ at each point in space), while the ratio $K_{1} / K_{2}$ varies in space in the form of two crossed saddle shapes, as in figure 1 . We assume that all consumers have identical (and fixed) maximum growth rates $G_{\max }$, mortality rates $m$, and diffusive dispersal rates $d$ but that their affinities to resources 1 and 2 can evolve within the constraints described below.

Consumers are characterized by a trait $\chi_{k}$, which describes a trade-off between affinities for the two resources as

$$
\begin{aligned}
& a_{1 k}=\frac{1}{1+e^{-\left(a_{0}+\chi_{k}\right)}}, \\
& a_{2 k}=\frac{1}{1+e^{-\left(a_{0}-\chi_{k}\right)}},
\end{aligned}
$$

pictured in figure $1 B$. Here $a_{0}$ is a trade-off control parameter that makes the trade-off strong for $a_{0}<0$, linear for $a_{0}=$ 0 , and weak for $a_{0}>0$. An example of a weak trade-off is shown in figure $1 B\left(a_{0}=2.5\right)$, which is the one we used in the numerical examples below (figs. 2, 3). The trade-off for the resource affinities is constructed in such a way that there exists a unique value of trait $\chi$ that maximizes consumer growth for each resource ratio when the trade-off is weak. Consumers with $\chi>0$ are better at acquiring resource 1 , and consumers with $\chi<0$ are better at acquiring resource 2 . Consumers with $\chi=0$ are perfect generalists. The landscape has reflective boundary conditions

$$
\begin{aligned}
& \left.\frac{\partial A_{k}}{\partial x}\right|_{x=-1, x=1}=0, \\
& \left.\frac{\partial A_{k}}{\partial y}\right|_{y=-1, y=1}=0,
\end{aligned}
$$


that is, consumers cannot disperse into or out of the landscape. The parameter values used in simulations are given in table 1.

To solve the ecological and evolutionary dynamics, we discretized the landscape to a $35 \times 35$ grid and used the methods described above in "Evolutionary Assembly in Continuous Space." Figure $2 A$ shows an example of an evolutionary process for the resource landscape depicted in figure $1 A$, where we seeded the landscape with a single phenotype and ended up with a community of 11 different consumers. Once an eco-evolutionary equilibrium has been reached, different consumer phenotypes will have settled onto spatial distributions reflective of their degree of specialization on either resource (fig. $2 C$ ), mirroring the distribution of the resource supply ratio (fig. 1A).

While the above findings are qualitatively intuitive, our approach greatly facilitates quantitative prediction of the exact number of evolving phenotypes, their trait values, population sizes, and spatial distributions. Note that quantitative prediction of these features is as easily achieved when spatial variation in local selection is highly irregular and thus precludes any intuition about even the qualitative nature of evolutionary outcomes. Quantitative prediction of evolutionary outcomes is a simple task only when the dispersal rate of consumers is either 0 (allowing perfect local adaptation) or very high (preventing local adaptation altogether; see below). In the following, we therefore explore in greater detail how these quantitative predictions depend on the consumers' rate of diffusion.

In a system that lacks spatial structure in resource supply, only one or two consumers can coexist when competing for two resources (sensu Tilman 1980). We observe the same phenomenon in the presence of spatial structure in resource supply when the diffusion rate of consumers is sufficiently high because the rate of diffusion controls to what extent consumers experience environmental heterogeneity. Figure 3 shows how the equilibrium distribution of trait values is affected by the rate of diffusion. When diffusion rates are sufficiently high all consumers more or less see only the average resource supply ratio, which is 1 . This means that only a single generalist consumer will exist, due to the weak trade-off between resource affinities. We established a cutoff at a rate of diffusion yielding 11 consumers since the computational complexity increases quite steeply with the number of consumers. In the limit of $d=0$, one would expect to have one consumer for each ratio $K_{1} / K_{2}$, since there exists a unique $\chi$ optimizing growth for each ratio, and with no diffusion to propagate the consumers each extant consumer will adapt to its local conditions.

To achieve a better understanding of the conditions that favor transition from a monomorphic to a polymorphic community, we employ the methods detailed in appendix B to determine the switch point between stabilizing and disruptive selection on a monomorphic consumer population for different diffusion rates. Due to the spatial symmetry of the supply ratios of resources $R_{1}$ and $R_{2}$ across the landscape, there is a spatially constant solution $A_{c}$ for the density of the monomorphic consumer at the evolutionarily singular point $\chi_{\mathrm{r}}=0$. We use this to analytically calculate an upper limit $C$ for the amount of disruptive $(C>0)$ or stabilizing $(C<0)$ selection (i.e., the second derivative of the invasion fitness with respect to the mutant trait):

$$
\begin{aligned}
C \leq & \left.\frac{1}{\int A_{c}^{2} \mathrm{~d} \mathbf{x}} \int A_{c}^{2} \frac{\partial^{2} G}{\partial \chi^{2}}\right|_{\chi=0} \mathrm{~d} \mathbf{x} \\
& +\frac{1}{\int A_{c}^{2} \mathrm{~d} \mathbf{x}} \int A_{c}^{2}\left(\left.\frac{\partial G}{\partial \chi}\right|_{\chi=0}\right)^{2} \mathrm{~d} \mathbf{x} \frac{L^{2}}{\pi^{2} d} .
\end{aligned}
$$

The first term is a weighted average of the curvatures of local selection, which here are downward concave (i.e., $\left.\left.\left(\partial^{2} G / \partial \chi^{2}\right)\right|_{\chi=0}<0\right)$ everywhere owing to the weak tradeoff in resource utilization. Due to the spatial symmetry in resource supply ratios, this results in stabilizing selection across the entire landscape: average fitness is highest for $\chi_{\mathrm{r}}=0$ because the local fitness gains of a phenotype with

\begin{tabular}{|c|c|c|c|}
\hline Quantity & Definition & Value/range & Unit \\
\hline$A_{k}$ & Density of consumer $k$ & $\ldots$ & mass area $^{-1}$ \\
\hline$R_{1,2}$ & Densities of resources 1 and 2 & $\ldots$ & mass area $^{-1}$ \\
\hline$m$ & Mortality rate & .1 & time $e^{-1}$ \\
\hline$d$ & Diffusion rate & $1.75 \cdot 10^{-6}$ & area time ${ }^{-1}$ \\
\hline$K_{1,2}$ & Maximum resource densities of resources 1 and 2 & See fig. $1 A$ & mass area $^{-1}$ \\
\hline$r_{1,2}$ & Renewal rates of resources 1 and 2 & 1 & time $^{-1}$ \\
\hline$a_{1 k, 2 k}$ & Resource affinity of consumer $k$ for resources 1 and 2 & $(0,1)$ & area mass ${ }^{-1}$ time $^{-1}$ \\
\hline$a_{0}$ & Trade-off strength control parameter & 2.5 & $\ldots$ \\
\hline$G_{\max }$ & Maximal consumer growth rate & 1 & time $^{-1}$ \\
\hline$\chi_{k}$ & Trait value for consumer $k$ & $(-\infty, \infty)$ & $\ldots$ \\
\hline$x$ & Horizontal spatial coordinate & $(-1,1)$ & length \\
\hline$y$ & Vertical spatial coordinate & $(-1,1)$ & length \\
\hline
\end{tabular}

Table 1: Parameters and state variables in example 1 
$\chi_{\mathrm{r}} \neq 0$ is outweighed by the inevitably higher local fitness losses in locations with the opposite resource supply ratios. The integral ratio in the second term describes a weighted variance of local directional selection. This ratio is multiplied by a measure of the size and shape of the landscape (here $L=2$ is the length of each side of the square landscape) and is divided by the diffusion rate $d$. As the average local selection is stabilizing (i.e., the first term in eq. [18] is negative), evolutionary branching can arise only from a sufficiently large ratio between the variance in local selection regimes and the diffusion rate. In this specific system, the solution for $A_{c}$ and $G$ does not depend on $d$, and we may thus set the above expression to 0 to calculate a diffusion rate above which we know that selection will be stabilizing. This calculation yields $d=3.86 \cdot 10^{-4}$, which agrees well with the value of $d$ at which the system transitions from monomorphic to polymorhpic (fig. 3).

\section{Example 2: Sinking Algae in a Water Column}

The previous example illustrated how phenotypic selection experienced by populations of randomly dispersing organisms depends both on the spatial pattern of local selection regimes and on the rate of dispersal across the landscape. In some systems, additional aspects of movement have to be taken into account to understand evolutionary responses to spatially varying selection. For example, many organisms disperse directionally in fluid media (e.g., streams, coastal longshore currents, wind) or by gravity. Such systems can be modeled with reaction-advection-diffusion equations where, in addition to the diffusion term, an advection term describing directional movement is introduced (Speirs and Gurney 2001; Huisman et al. 2002; Anderson et al. 2005).

This directional movement presents a new type of problem. The necessary technical condition allowing us to compute the expression for the selection gradient (eq. [10]) is that the differential operator describing the dynamics is what is known as "self-adjoint" (for details, see app. A). Intuitively speaking, this condition, in the spatial case, means that the dispersal or transport rate from a coordinate $x$ to a coordinate $y$ is equal to that from $y$ to $x$. In the discrete spatial case, this condition is equivalent to having a symmetric dispersal matrix. In the continuous case, this imposes conditions both on the form of the operator itself and on the boundary conditions of the system. Advection breaks this symmetry, but for the case of constant advection this issue can be resolved by means of a variable transform (app. E), which is what we have applied in the example below.

An example of a widespread ecological system described by a reaction-advection-diffusion equation is sinking plankton algae growing in a pelagic environment. The spatial dynamics of this system are considerably more complex than those of example 1 because, in addition to the added com- plication of directional algal movement, resources are also transported through space by either turbulent diffusion (dissolved nutrients) or directional flux (light). Such systems are typically characterized by opposing vertical resource gradients (with light availability decreasing and nutrient availability increasing with depth), setting up a smooth, continuous gradient of spatially varying selection for light versus nutrient use capacities. As an illustration, we use the so-called fixed stoichiometry version of a phytoplankton model by Jäger et al. (2010), where nutrients from algae that have settled out of the water column are recycled in the bottom sediment. The equations that describe the ecological dynamics are reproduced in appendix $\mathrm{E}$ together with the details of how the evolutionary dynamics were implemented.

The growth rate of the algae is proportional to the product $R /\left(M_{j}+R\right) \cdot I /\left(H_{j}+I\right)$ of two Monod functions describing the dependence of algal growth on available nutrients $(R)$ and light $(I)$. We introduce evolutionary dynamics to the system by letting the half-saturation constants of phenotype $j$ for nutrient and light uptake $M_{j}$ and $H_{j}$ depend on a trait $\chi_{j}$ such that $M_{j}=M_{0} / \chi_{j}$ and $H_{j}=H_{0} \chi_{j}$. This implies a weak trade-off between the half-saturation constants, where phenotypes with a high value of $\chi$ are good nutrient competitors and phenotypes with a low value of $\chi$ are good light competitors (fig. $4 A$ ).

To study the evolutionary consequences of directional dispersal-in this case, sinking - we considered a 50-m-deep water column and let the system run to eco-evolutionary equilibrium (in the same way as in example 1) for a range of algal sinking speeds, $v$. For the chosen parameter values, sinking speed affects not only the equilibrium distribution of phenotype trait values but also the number of coexisting phenotypes in a nonmonotonic manner (fig. 4B). With increasing sinking speed, the phenotypes' depth distributions become centered at increasingly greater depths (fig. $4 \mathrm{C}$, $4 D)$, and the overall biomass in the system generally decreases (fig. $4 E$ ). For the investigated parameter range, a strong light competitor, pictured in green in figure 4 , tends to dominate the system.

It is far from obvious why intermediate sinking speeds generate the most diverse communities. One possible explanation could be that only at intermediate rates of sinking does the depth separation between the top and bottom phenotypes become large enough to admit an intermediate phenotype (see fig. $4 F$ ). We limited our investigations to a realistic range of sinking speeds $v$ for which we observed equilibrium dynamics and for which the numerical approximation of the transformation of equation (9) (app. E) was accurate at the implemented spatial resolution. When sinking speeds are increased further, the system first moves into limit cycle dynamics until, at around $v=4 \mathrm{~m} \mathrm{day}^{-1}$, sinking losses become so large that no population is viable. 
A

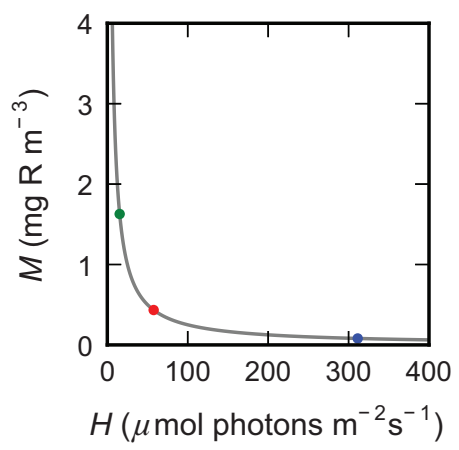

B

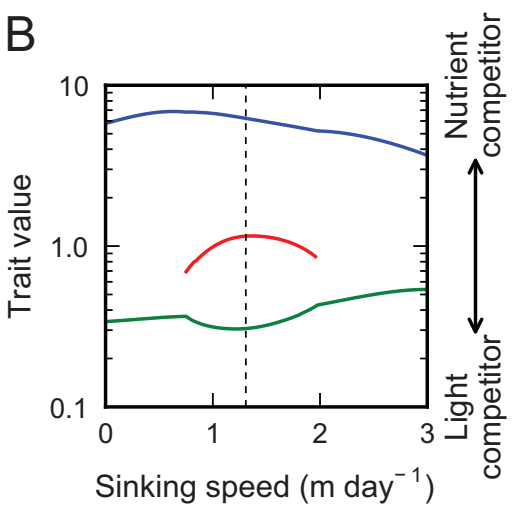

$\mathrm{E}$

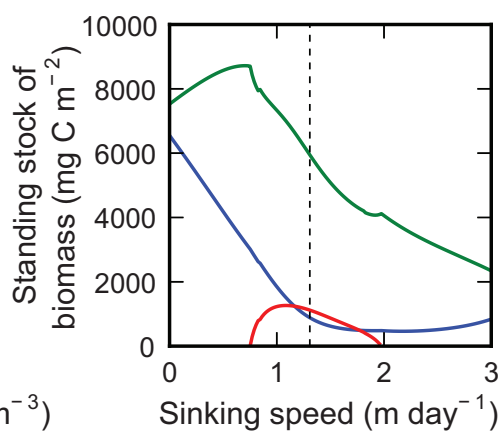

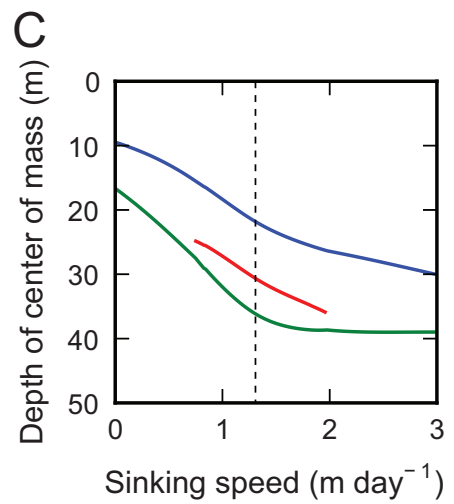

$\mathrm{F}$

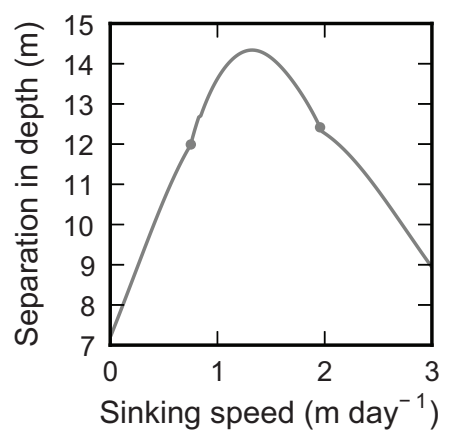

Figure 4: Example 2. A, Trade-off between the half-saturation constants for light $(H)$ and nutrient $(M)$ uptake. The three dots show the combination of half-saturation constants for the three phenotypes at the sinking speed indicated by the dashed lines in $B, C$, and $E$. $B$, Ecoevolutionary equilibrium distribution of trait values for different sinking speeds. Phenotypes with low trait values are good light competitors, and phenotypes with high trait values are good nutrient competitors. $C$, Depth of the center of mass of the biomass depth distributions for different sinking speeds. $D$, Depth distribution of biomass concentration of the three phenotypes at the sinking speed indicated by the dashed lines in $B, C$, and $E$. E, Standing stock of biomass of the algal phenotypes for different sinking speeds. The standing stock of a phenotype is its biomass concentration integrated over the entire water column. $F$, Depth separation between the center of mass of the top and bottom phenotypes (blue and green in $A-E$ ). The dots delimit the range of intermediate sinking speeds over which the third (red) phenotype is part of the ensemble. In all panels, different colors represent different phenotypes, with the color codes being consistent among panels.

\section{Computational Benefits}

Numerically, the main advantage of using the perturbation expression for calculating selection gradients, equation (10), is that it does not rely on any explicit computations of eigenvalues or eigenfunctions. If the expression were not available, the selection gradient would have to be estimated numerically by calculating, for example, $\left[\lambda_{\mathrm{d}}\left(\chi_{\mathrm{r}}+\epsilon\right)-\right.$ $\left.\lambda_{\mathrm{d}}\left(\chi_{\mathrm{r}}-\epsilon\right)\right] /(2 \epsilon)$ for some small number $\epsilon$, which would require the numerical calculation of the dominant eigenvalue of $F\left(\chi_{\mathrm{r}}+\epsilon\right)$ and $F\left(\chi_{\mathrm{r}}-\epsilon\right)$. Numerical calculation of eigenvalues of large matrices is, however, time-consuming, and hence using equation (10) can yield very significant computational time savings. This is particularly true when setting the rate of change of a trait in time to be proportional to the selection gradient, in which case many evaluations of the selection gradient have to be made. As an example, we timed the calculations of the selection gradients for all residents in figure 3. The average time for numerical computation of the selection gradient using Matlab's (R2014a) eigs function was $0.102 \mathrm{~s}$, compared with $6.03 \cdot 10^{-5} \mathrm{~s}$ for the perturbation calculation. This makes the perturbation calculation more than 1,500 times faster. Using the above estimates of average computation times and (conservatively) assuming that the selection gradient has to be calculated only once per resident and time step, calculation of all selection gradients needed to produce figure $2 \mathrm{~A}$ would have taken over $10 \mathrm{~h}$ without the perturbation methods but takes only roughly $21 \mathrm{~s}$ with them.

\section{Discussion}

\section{Contributions to Understanding Selection in Space}

Apart from their use as efficient tools for modeling specific evolutionary scenarios, the expressions derived for di- 
rectional and stabilizing/disruptive selection yield several novel, general insights. For example, when the trait under selection does not affect movement, equation (11) shows that the selection gradient in the spatial system can be understood as a weighted average of local selection gradients with weights proportional to the square of the resident population's equilibrium distribution. Equation (11) can thus in principle be used to predict the direction of evolution of a trait under selection in a heterogeneous environment. This would require accurate measurements of local population densities and of how per capita growth rates change with trait values (i.e., of $\partial G / \partial \chi$ ). The latter is a challenging and labor-intensive task in most natural systems. An alternative would be to investigate to what extent selection gradients measured using the approach of Lande and Arnold (1983) could serve as useful proxies for $\partial G / \partial \chi$, as there is already a wealth of such measurements (see, e.g., Siepielski et al. 2013 and references therein).

Furthermore, equation (11) makes a contribution to the unresolved issue of widespread local directional selection combined with evolutionary stasis (Merilä et al. 2001). Specifically, the expression shows that even if the population-level selection gradient is 0 , as in evolutionary stasis, local measurements of the selection gradient will very likely be nonzero and point in different directions at different points in a heterogeneous environment. While this possibility has been conjectured previously, equation (11) lends mathematical support to it and could be used to ascertain to what degree space is responsible for the directional selection/stasis dichotomy in real populations.

Finally, when it comes to disruptive selection in heterogeneous landscapes, the methods described herein (for details, see app. B) can be used to determine whether the source of disruptive selection is a global trade-off across the phenotype range in, for example, physiology or behavior or the environmental heterogeneity of selective regimes prompting local adaptations. This was shown in example 1, where the spatially averaged stabilizing trade-off in resource utilization could be overcome by disruptive selection contributed by variability in selection regimes provided that the rate of dispersal was sufficiently low. Depending on which type of disruptive selection is the primary contributor, one may forecast on a rough level the degree to which the branched phenotypes can be expected to have spatial extensions similar to those of their progenitor. Furthermore, if measurements of $\partial^{2} G / \partial \chi^{2}$ could be obtained in addition to the local selection gradients, equation (B7) could be used to rule out disruptive selection in the same way we analyzed example 1.

\section{Limitations of Reaction-Diffusion Approaches}

Reaction-diffusion models are not always appropriate for describing ecological dynamics. In particular, the discrete nature of individuals is not modeled, and the diffusion operator instantaneously propagates infinitesimally low densities across any region. This may lead to some pathological behavior, such as the somewhat infamous atto-fox (Mollison 1991), where $10^{-18}$ of an infected fox causes a reinvasion of rabies. Reaction-diffusion equations furthermore do not always replicate the limiting behavior of an underlying stochastic spatial process, as pointed out by Durrett and Levin (1994), although the authors remark that this issue can sometimes be alleviated by correcting the reaction terms by deriving them directly from the stochastic process. The shortcomings of reaction-diffusion equations thus practically invalidate their use for studying evolution in settings where mutant-mutant interactions or limited movement are critical to the outcome. Examples include the evolution of cooperation, where spatial structure has been shown to allow local clusters of cooperators to invade a community of defectors (for perspectives on these shortcomings, see, e.g., Ferrière and Le Galliard 2001; Doebeli and Dieckmann 2003; Mágori et al. 2005; Lion and van Baalen 2008). Before applying the framework developed in this article, one should therefore carefully assess that neither the ecological nor the evolutionary processes of interest are critically dependent on the discrete nature of individuals or on limited dispersal rates of mutants.

Although there is no straightforward way of taking local mutant-mutant interactions into account in reactiondiffusion models, as in other frameworks (see the next section), a possible way of modeling limited dispersal in reaction-diffusion systems is with nonlinear diffusion terms. Using so-called slow diffusion, the spread of infinitesimal densities is no longer instantaneous. Such approaches have occasionally been used to model the ecology of populations, for example, of microorganisms (Eberl et al. 2001; Tao and Winkler 2013) and insects (Turchin 1989). Using nonlinear diffusion to model spread implies that parts of a landscape may become inaccessible to some of the phenotypes. This in turn suggests that the location of invasion of a mutant would be critical to its success, as opposed to linear diffusion models, where the mutant spreads globally while still rare. An adaptive dynamics framework for these types of nonlinear diffusion equations does, however, not yet exist, and the formulation and analysis of such a framework would be a natural continuation of the material presented here.

\section{Other Analytical Approaches to Modeling Spatial Evolution}

As the eco-evolutionary dynamics of spatially structured systems can be very complicated, some realism must inevitably be sacrificed to successfully derive analytical insights. Depending on the focus of interest, different approaches 
have been developed to handle specific phenomena while neglecting other parts of ecological, evolutionary, or spatial dynamics for analytical feasibility.

For example, starting with Wright (1943) there has been a large body of population-genetics theory aiming at understanding genetic structure in finite, or locally finite, populations (for a review, see Rousset 2004). The framework considers dispersing individuals on a possibly infinite set of discrete patches, where the expected allele frequencies are tracked. These models have the advantage that they enable the study of both genetically explicit selection through a weak selection approximation and the effects of genetic drift. Incorporating demographic dynamics into these models is, however, very complicated relative to phenotypic approaches, such as adaptive dynamics or quantitative genetics, and most models simply consider population sizes to be constant (but see, e.g., Rousset and Ronce 2004). Models of this type may also be used to investigate the evolution of helping behaviors (Rousset 2004; Lehmann et al. 2006), as they can take local mutant-mutant interactions into account.

Another approach to studying phenotypic evolution in spatially structured systems is to use adaptive dynamics techniques coupled with moment-based approximations (van Baalen and Rand 1998; Lion and van Baalen 2008; Lion 2015). Typically, the demographic dynamics of individualbased lattice models are approximated by deriving equations for the temporal change in the density of individuals (first moment) and of pairs (second moment), after which the moment hierarchy is "closed" by replacing higherorder moments, such as the density of triplets, with expressions based on lower-order moments. These methods have worked well for studying evolutionary processes in space where local mutant-mutant interactions are critical, such as the evolution of helping behaviors (van Baalen and Rand 1998; Le Galliard et al. 2003; Ohtsuki et al. 2006; Lion and van Baalen 2009). A corresponding approximation method for populations in continuous space has been developed (Bolker and Pacala 1997; Law and Dieckmann 2000) and should be applicable to evolutionary studies (a somewhat different moment-based method has already been applied by North et al. [2011]). Underlying these techniques is the implicit assumption that space, at least in a statistical sense, looks the same from the perspective of any focal individual. They therefore work best when the underlying environment is homogeneous and are thus hard to apply to cases like our example 2.

\section{Conclusions and Outlook}

While there will always be exceptions, we believe the following advice can be given for modeling phenotypic evolution of spatially structured populations: If the primary goal of the study is to understand how local individual interactions evolve or how evolution acts near to a mean-field limit, moment-based methods are appropriate. If the goal is to understand intraspecific variation in a trait throughout heterogeneous space, then reaction-diffusion quantitative genetics models are best suited. Finally, if the goal is to study the evolution of a spatially constant trait in a heterogeneous environment, especially for finding evolutionarily stable communities or studying disruptive selection, then the methods detailed in this article constitute the best alternative.

Selection in space continues to be a challenging problem, with no single theoretical framework striking the balance between tractability and insight on the one hand and scope and realism on the other. We believe that this article will provide the groundwork for using reaction-diffusion equations coupled with adaptive dynamics to answer questions about selection in space that were previously difficult to address.

\section{Acknowledgments}

A.B., S.D., and J.W. gratefully acknowledge support from the Swedish Research Council (2015-3917). We thank S. Geritz, J. Ripa, and two anonymous reviewers for valuable comments that helped to greatly improve the article. B.B. received support from Deutsche Forschungsgemeinschaft (DFG) priority program 1704 (DynaTrait).

\section{Literature Cited}

Ajar, É. 2003. Analysis of disruptive selection in subdivided populations. BMC Evolutionary Biology 3:22.

Anderson, K. E., R. M. Nisbet, S. Diehl, and S. D. Cooper. 2005. Scaling population responses to spatial environmental variability in advection-dominated systems. Ecology Letters 8:933-943.

Barton, N. H. 1983. Multilocus clines. Evolution 37:454-471.

. 1999. Clines in polygenic traits. Genetical Research 74:223236.

Birand, A., A. Vose, and S. Gavrilets. 2012. Patterns of species ranges, speciation, and extinction. American Naturalist 179:1-21.

Blondel, J., D. W. Thomas, A. Charmantier, P. Perret, P. Bourgault, and M. M. Lambrechts. 2006. A thirty-year study of phenotypic and genetic variation of blue tits in Mediterranean habitat mosaics. Bioscience 56:661-673.

Bolker, B., and S. W. Pacala. 1997. Using moment equations to understand stochastically driven spatial pattern formation in ecological systems. Theoretical Population Biology 52:179-197.

Britton, N. F. 1986. Reaction-diffusion equations and their applications to biology. Academic Press, London.

Cantrell, R. S., and C. Cosner. 2004. Spatial ecology via reactiondiffusion equations. Wiley, Chichester.

Case, T. J., and M. L. Taper. 2000. Interspecific competition, environmental gradients, gene flow, and the coevolution of species' borders. American Naturalist 155:583-605.

Caswell, H. 2001. Matrix population models. 2nd ed. Sinauer, Sunderland, MA. 
Champagnat, N. 2003. Convergence of adaptive dynamics $n$-morphic jump processes to the canonical equation and degenerate diffusion approximation. Prépublication de l'Université de Nanterre (Paris X) No. 3.

Day, T. 2001. Population structure inhibits evolutionary diversification under competition for resources. Genetica 112:71-86.

Dieckmann, U., and R. Law. 1996. The dynamical theory of coevolution: a derivation from stochastic ecological processes. Journal of Mathematical Biology 34:579-612.

Dockery, J., V. Hutson, K. Mischaikow, and M. Pernarowski. 1998. The evolution of slow dispersal rates: a reaction diffusion model Journal of Mathematical Biology 37:61-83.

Doebeli, M., and U. Dieckmann. 2003. Speciation along environmental gradients. Nature 421:259-264.

Durrett, R., and S. Levin. 1994. The importance of being discrete (and spatial). Theoretical Population Biology 46:363-394.

Eberl, H. J., D. F. Parker, and M. Van Loosdrecht. 2001. A new deterministic spatio-temporal continuum model for biofilm development. Computational and Mathematical Methods in Medicine 3: 161-175.

Eldredge, N., J. N. Thompson, P. M. Brakefield, S. Gavrilets, D. Jablonski, J. B. Jackson, R. E. Lenski, B. S. Lieberman, M. A. McPeek, and W. Miller III. 2005. The dynamics of evolutionary stasis. Paleobiology 31:133-145.

Felsenstein, J. 1976. The theoretical population genetics of variable selection and migration. Annual Review of Genetics 10:253-280.

Ferrière, R., and J.-F. Le Galliard. 2001. Invasion fitness and adaptive dynamics in spatial population models. Pages 57-79 in J. Clobert, E. Danchin, A. A. Dhondt, and J. D. Nichols, eds. Dispersal. Oxford University Press, New York.

Fisher, R. A. 1937. The wave of advance of advantageous genes. Annals of Eugenics 7:355-369.

. 1950. Gene frequencies in a cline determined by selection and diffusion. Biometrics 6:353-361.

Gavrilets, S., and A. Vose. 2005. Dynamic patterns of adaptive radiation. Proceedings of the National Academy of Sciences of the USA 102:18040-18045.

Geritz, S., E. Kisdi, G. Meszéna, and J. Metz. 1998. Evolutionarily singular strategies and the adaptive growth and branching of the evolutionary tree. Evolutionary Ecology 12:35-57.

Haldane, J. 1948. The theory of a cline. Journal of Genetics 48:277-284.

Haller, B. C., R. Mazzucco, and U. Dieckmann. 2013. Evolutionary branching in complex landscapes. American Naturalist 182:E127E141.

Hastings, A. 1983. Can spatial variation alone lead to selection for dispersal? Theoretical Population Biology 24:244-251.

Holmes, E. E., M. A. Lewis, J. Banks, and R. Veit. 1994. Partial differential equations in ecology: spatial interactions and population dynamics. Ecology 75:17-29.

Huisman, J., M. Arrayás, U. Ebert, and B. Sommeijer. 2002. How do sinking phytoplankton species manage to persist? American Naturalist 159:245-254.

Jäger, C. G., S. Diehl, and M. Emans. 2010. Physical determinants of phytoplankton production, algal stoichiometry, and vertical nutrient fluxes. American Naturalist 175:E91-E104.

Kingsolver, J. G., and S. E. Diamond. 2011. Phenotypic selection in natural populations: what limits directional selection? American Naturalist 177:346-357.

Kirkpatrick, M., and N. H. Barton. 1997. Evolution of a species' range. American Naturalist 150:1-23.
Kooijman, S. 1998. The synthesizing unit as model for the stoichiometric fusion and branching of metabolic fluxes. Biophysical Chemistry 73:179-188.

Kubisch, A., R. D. Holt, H.-J. Poethke, and E. A. Fronhofer. 2014. Where am I and why? synthesizing range biology and the ecoevolutionary dynamics of dispersal. Oikos 123:5-22.

Lande, R. 1979. Quantitative genetic analysis of multivariate evolution, applied to brain: body size allometry. Evolution 33:402-416.

Lande, R., and S. J. Arnold. 1983. The measurement of selection on correlated characters. Evolution 37:1210-1226.

Law, R., and U. Dieckmann. 2000. Moment approximations of individual-based models. Pages 252-270 in U. Dieckmann, R. Law, and J. Metz, eds. The geometry of ecological interactions: simplifying spatial complexity. Cambridge University Press, Cambridge.

Le Galliard, J.-F., R. Ferrière, and U. Dieckmann. 2003. The adaptive dynamics of altruism in spatially heterogeneous populations. Evolution 57:1-17.

Lehmann, L., N. Perrin, and F. Rousset. 2006. Population demography and the evolution of helping behaviors. Evolution 60:1137-1151.

Lion, S. 2015. Moment equations in spatial evolutionary ecology. Journal of Theoretical Biology 405:46-57.

Lion, S., and M. van Baalen. 2008. Self-structuring in spatial evolutionary ecology. Ecology Letters 11:277-295.

2009. The evolution of juvenile-adult interactions in populations structured in age and space. Theoretical Population Biology 76:132-145.

Mágori, K., P. Szabó, F. Mizera, and G. Meszéna. 2005. Adaptive dynamics on a lattice: role of spatiality in competition, co-existence and evolutionary branching. Evolutionary Ecology Research 7:121.

Merilä, J., B. Sheldon, and L. Kruuk. 2001. Explaining stasis: microevolutionary studies in natural populations. Genetica 112:199222.

Metz, J., R. Nisbet, and S. Geritz. 1992. How should we define "fitness" for general ecological scenarios? Trends in Ecology and Evolution 7:198-202.

Mizera, F., and G. Meszéna. 2003. Spatial niche packing, character displacement and adaptive speciation along an environmental gradient. Evolutionary Ecology Research 5:1-20.

Mollison, D. 1991. Dependence of epidemic and population velocities on basic parameters. Mathematical Biosciences 107:255-287.

Monod, J. 1949. The growth of bacterial cultures. Annual Reviews in Microbiology 3:371-394.

Nagylaki, T. 1975. Conditions for the existence of clines. Genetics 80:595-615.

Norberg, J., M. C. Urban, M. Vellend, C. A. Klausmeier, and N. Loeuille. 2012. Eco-evolutionary responses of biodiversity to climate change. Nature Climate Change 2:747-751.

North, A., S. Cornell, and O. Ovaskainen. 2011. Evolutionary responses of dispersal distance to landscape structure and habitat loss. Evolution 65:1739-1751.

Ohtsuki, H., C. Hauert, E. Lieberman, and M. A. Nowak. 2006. A simple rule for the evolution of cooperation on graphs and social networks. Nature 441:502-505.

Press, W. H., S. A. Teukolsky, W. T. Vetterling, and B. P. Flannery. 2007. Numerical recipes: the art of scientific computing. 3rd ed. Cambridge University Press, Cambridge.

Rousset, F. 2004. Genetic structure and selection in subdivided populations. Monographs in Population Biology No. 40. Princeton University Press, Princeton, NJ. 
Rousset, F., and O. Ronce. 2004. Inclusive fitness for traits affecting metapopulation demography. Theoretical Population Biology 65 127-141.

Servedio, M. R., and S. Gavrilets. 2004. The evolution of premating isolation: local adaptation and natural and sexual selection against hybrids. Evolution 58:913-924.

Siepielski, A. M., K. M. Gotanda, M. B. Morrissey, S. E. Diamond, J. D. DiBattista, and S. M. Carlson. 2013. The spatial patterns of directional phenotypic selection. Ecology Letters 16:1382-1392.

Slatkin, M. 1973. Gene flow and selection in a cline. Genetics 75:733756.

. 1975. Gene flow and selection in a two-locus system. Genetics 81:787-802.

. 1978. Spatial patterns in the distributions of polygenic characters. Iournal of Theoretical Biology 70:213-228.

Speirs, D. C., and W. S. Gurney. 2001. Population persistence in rivers and estuaries. Ecology 82:1219-1237.

Tao, Y., and M. Winkler. 2013. Locally bounded global solutions in a three-dimensional chemotaxis-stokes system with nonlinear diffusion. Annales de l'Institut Henri Poincaré (C) Analvse Non Linéaire 30:157-178.

Thompson, J. N. 1999. Specific hypotheses on the geographic mosaic of coevolution. American Naturalist 153:S1-S14.

Tilman, D. 1980. Resources: a graphical-mechanistic approach to competition and predation. American Naturalist 116:362-393.

- 1982. Resource competition and community structure. Monographs in Population Biology. Princeton University Press, Princeton, NJ.

Troost, T. A., B. W. Kooi, and S. A. L. M. Kooijman. 2005. Ecological specialization of mixotrophic plankton in a mixed water column. American Naturalist 166:E45-E61.

Turchin, P. 1989. Population consequences of aggregative movement. Lournal of Animal Ecology 58:75-100.

van Baalen, M., and D. A. Rand. 1998. The unit of selection in viscous populations and the evolution of altruism. Lournal of Theoretical Biology 193:631-648.
Waxman, D., and S. Gavrilets. 2005. Issues of terminology, gradient dynamics and the ease of sympatric speciation in adaptive dynamics. Journal of Evolutionary Biology 18:1214-1219.

Wright, S. 1943. Isolation by distance. Genetics 28:114-138.

\section{References Cited Only in the Online Appendixes}

de Mazancourt, C., and U. Dieckmann. 2004. Trade-off geometries and frequency-dependent selection. American Naturalist 164:765778.

Durán, R. G., and M. A. Muschietti. 2001. An explicit right inverse of the divergence operator which is continuous in weighted norms. Studia Mathematica 148:207-219.

Evans, L. C. 2010. Partial differential equations. 2nd ed. American Mathematical Society, Providence, RI.

Feynman, R. 1939. Forces in molecules. Physical Review 56:340.

Geritz, S. A., E. van der Meijden, and J. A. Metz. 1999. Evolutionary dynamics of seed size and seedling competitive ability. Theoretical Population Biology 55:324-343.

Hellmann, H. 1933. Zur rolle der kinetischen elektronenenergie für die zwischenatomaren kräfte. Zeitschrift für Physik 85:180-190.

Pechstein, C., and R. Scheichl. 2011. Weighted Poincaré inequalities and applications in domain decomposition. Pages 197-204 in Y. Huang, R. Kornhuber, R. Widlund, and J. Xu. Domain decomposition methods in science and engineering XIX. Springer, Berlin.

Ryabov, A., and B. Blasius. 2008. Population growth and persistence in a heterogeneous environment: the role of diffusion and advection. Mathematical Modelling of Natural Phenomena 3:42-86.

Sakurai, J. J., and S. F. Tuan. 1985. Modern quantum mechanics. Vol. 1. Addison-Wesley, Reading, MA.

Associate Editor: Scott L. Nuismer Editor: Yannis Michalakis

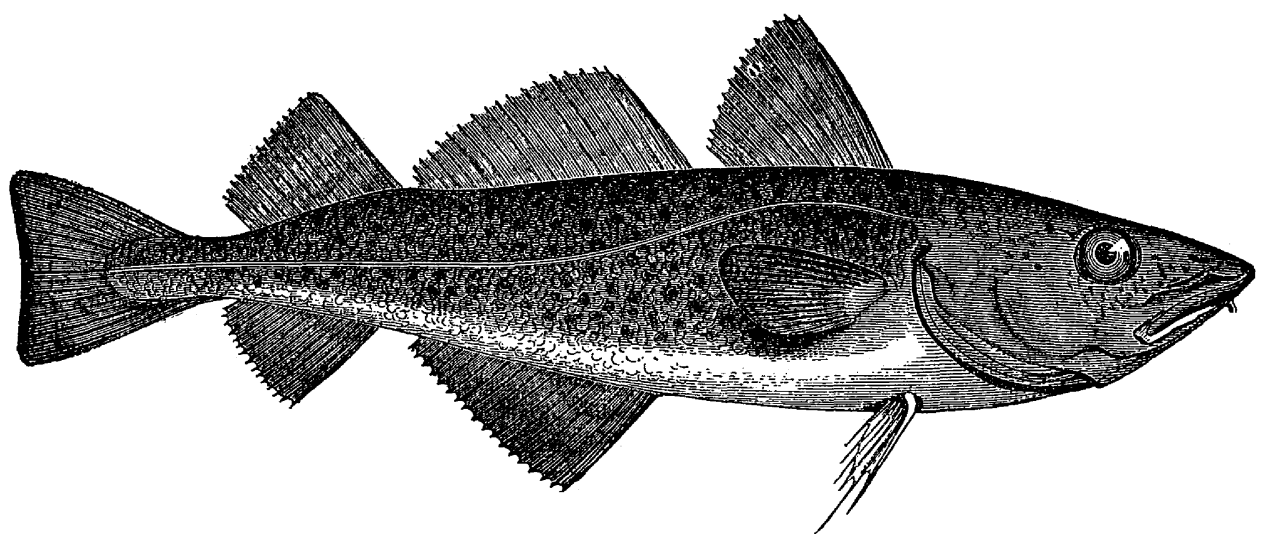

"The Codfish (Morrhua Americana). . . is found in our markets all the year, but is not taken at all times from the same locality or fishingground. This fish does not migrate along the coast, but acquires its desired temperature by gradually moving from shallower to deeper water, and returning as the season grows colder." From "The Habits and Migrations of Some of the Marine Fishes of Massachusetts" by James H. Blake (The American Naturalist, 1870, 4:513-521). 